\title{
ON EXISTENCE OF OSCILLATORY SOLUTIONS FOR A SECOND ORDER SUBLINEAR DIFFERENTIAL EQUATION
}

\author{
JAMES S. W. WONG \\ In Memory of Zeev Nehari
}

\begin{abstract}
A result on the existence of oscillatory solutions for the second order sublinear differential equation $y^{\prime \prime}+a(t)|y|^{\gamma} \operatorname{sgn} y=0,0<\gamma<1$, where $a(t)$ is positive and continuous, is given. This supplements a well-known result of Hinton for the superlinear case, i.e. $\gamma>1$.
\end{abstract}

1. We consider the second order Emden-Fowler equation

$$
y^{\prime \prime}(t)+a(t)|y(t)|^{\gamma} \operatorname{sgn} y(t)=0,
$$

on $[0, \infty)$, where $a(t)$ is a positive continuous function. We are here concerned with the so-called sublinear case of (1), i.e. when $0<\gamma<1$. On the other hand, equation (1) is called superlinear when $\gamma>1$. It is known in the sublinear case that every solution is continuable throughout $[0, \infty)$ and under the additional assumption that $a(t)$ is locally of bounded variation on $[0, \infty)$, then (1) has a unique solution satisfying every set of prescribed initial conditions. Hence, under these assumptions, which we make throughout, the set of zeros of any particular solution of $(1)$ on $[0, \infty)$ is either finite or infinite extending to infinity with no accumulation points. In fact, the assumptions made on $a(t)$ are sufficient also to ensure that the solutions of the superlinear equation $(1), \gamma>1$, have the same properties (see e.g. $[4,21])$. Equation (1) is said to be oscillatory if every solution has infinitely many zeros and it is said to be nonoscillatory if every solution has only a finite number of zeros. However, for nonlinear equation (1), when $\gamma \neq 1$, there may exist oscillatory and nonoscillatory solutions, a fact precluded by the Sturm's separation theorem for the linear equation (1), when $\gamma=1$.

Earlier works of Coffman and Wong $[\mathbf{3}, \mathbf{4}]$ and more recently Wong $[\mathbf{2 1}, \mathbf{2 2}]$, Kwong and Wong [14] and Erbe [5], Erbe and Muldowney [6] have discussed the concept of a "duality principle" between sublinear $(0<\gamma<1)$ and superlinear $(\gamma>1)$ equations, whereby results concerning oscillation and nonoscillation of solutions in one case would lead to a similar result in the other. For oscillation theorems and existence of nonoscillatory solutions, this was illustrated in [3], and for nonoscillation theorems, we refer to $[4,14,22]$. In both of these two categories of theorems, the dual result was obtained by setting $\gamma=1$ in one of such oscillation and nonoscillation criteria as the case may be.

In the more delicate cases of existence of oscillatory or nonoscillatory solutions, i.e. conditions on $a(t)$ not sufficient to imply all solutions be oscillatory or nonoscillatory but enough to guarantee the existence of at least one oscillatory or nonoscillatory solution, the "duality principle" has not been adequately illustrated. The

Received by the editors August 31, 1983.

1980 Mathematics Subject Classification. Primary 34C10, 34C15.

Key words and phrases. Second order, nonlinear, differential equations, oscillation.

(C) 1984 American Mathematical Society $0002-9939 / 84 \$ 1.00+\$ .25$ per page 
results of $[\mathbf{2}, \mathbf{9}-\mathbf{1 1}, \mathbf{1 7}]$, as extended by Erbe and Muldowney [6] seem to indicate that oscillation criteria of this kind remain in the same form for both sublinear and superlinear equations. In particular, the following is known:

Theorem A (ERbe And Muldowney [6]). Let $\gamma>0$. Assume that

$$
t^{(\gamma+3) / 2} a(t)(\log t)^{\sigma}
$$

is nondecreasing for some $\sigma \leq 0$. Then equation (1) has oscillatory solutions.

Corresponding result to Theorem A for all solutions of (1) to be nonoscillatory, remains elusive, see the recent article by Erbe and Muldowney [7] and also Erbe $[5]$.

The purpose of this note is to prove the sublinear analogue of a result of Hinton [8] on the existence of oscillatory solution which involves integral conditions on $a(t)$ rather than monotoneity conditions. We prove the following result:

THEOREM B. Let $0<\gamma<1$ and let $\varphi(t)=a^{-1 /(\gamma+3)}(t)$. Assume that

$$
\int^{\infty} \frac{d t}{\varphi^{2}(t)}=\infty
$$

and

$$
\int^{\infty}\left|\varphi \varphi^{\prime \prime}(t)\right| d t<\infty
$$

then equation (1) has oscillatory solutions.

Hinton [8] showed that Theorem $B$ is valid both in the superlinear and linear cases, i.e. when $\gamma \geq 1$. Thus from Theorems $\mathrm{A}$ and $\mathrm{B}$, we discover that criteria ensuring the existence of oscillatory solutions remain in the same form for both superlinear and sublinear equations.

2. To prove Theorem $\mathrm{B}$, let $y(t)$ be any nonoscillatory solution which may be assumed without loss of generality positive for $t \geq t_{0}>0$. Let $Z(t)=[y(t) / \varphi(t)]^{\gamma}$, where $\varphi(t)$ is defined as in Theorem B. Equation (1) implies that $Z(t)$ satisfies the second order nonlinear equation (here $\beta=1 / \gamma>1$ ):

$$
\left(\varphi Z^{\beta-1}\right)^{\prime \prime}+(\beta-1) \varphi Z^{\beta-3} Z^{\prime 2}+\left(-\frac{1}{\beta}\right) \varphi^{\prime \prime} Z^{\beta-1}+(1-\gamma) a \psi^{\gamma}=0,
$$

which was found very useful in establishing oscillation theorems for sublinear equations, $[\mathbf{1 1}, \mathbf{1 3}, \mathbf{1 5}, \mathbf{1 9}]$. Multiplying (4) through by $\varphi$ and integrating, we find

$$
\begin{gathered}
\varphi^{2}\left(Z^{\beta-1}\right)^{\prime}(t)+(1-\gamma) \int_{t_{0}}^{t} Z^{\beta-1} \varphi \varphi^{\prime \prime}+(\beta-1) \int_{t_{0}}^{t} \varphi^{2} Z^{\beta-3} Z^{\prime 2} \\
+(1-\gamma) \int_{t_{0}}^{t} a \varphi^{\gamma+1}=c_{0}=\varphi^{2}\left(Z^{\beta-1}\right)^{\prime}\left(t_{0}\right) .
\end{gathered}
$$

Since the second integral is nonnegative and if we assume $Z(t)$ is bounded, say by $M$, then (5) can be estimated as follows

$$
\varphi^{2}\left(Z^{\beta-1}\right)^{\prime}(t) \leq c_{0}+(1-\gamma)\left\{M^{\beta-1} \int_{t_{0}}^{t}\left|\varphi \varphi^{\prime \prime}\right|-\int_{t_{0}}^{t} a \varphi^{\gamma+1}\right\} .
$$


Note that $a \varphi^{\gamma+1}=\varphi^{-2}$; hence by (2) and (3) the right-hand side of (6) becomes eventually negative. Indeed, there exists $t_{1} \geq t_{0}$ such that for $t \geq t_{1}$, we can have

$$
\varphi^{2}\left(Z^{\beta-1}\right)^{\prime}(t) \leq-1, \quad t \geq t_{1} .
$$

Dividing (7) by $\varphi^{2}$ and integrating once more, we obtain

$$
z^{\beta-1}(t) \leq Z^{\beta-1}\left(t_{1}\right)-\int_{t_{1}}^{t} \frac{d s}{\varphi^{2}(s)},
$$

which implies that $Z(t)$ must eventually be negative, contradicting the fact that $y(t)>0$.

We now complete the proof by showing the existence of a certain solution $y(t)$ of equation (1) such that $Z(t)=[y(t) / \varphi(t)]^{\gamma}$ is bounded. We follow a similar argument employed by Hinton [8]. Define

$$
V(s)=\frac{1}{2} x^{2}(s)+\frac{x^{\gamma+1}(s)}{\gamma+1},
$$

where $x(s)=y(t) / \varphi(t)=Z(t)^{\beta}$ and $s=\int_{0}^{t} d \sigma / \varphi^{2}(\sigma)=h(t)$. Using (1), we differentiate (8) to obtain

$$
V^{\prime}(s)=-\left[\varphi^{3} \varphi^{\prime \prime}(t)\right] x(s) x^{\prime}(s) .
$$

From (8), we have $\left|x^{\prime}(s)\right| \leq \sqrt{2} V^{1 / 2}(s),|x(s)| \leq K V^{1 /(\gamma+1)}(s)$, where $K^{\gamma+1}=$ $1 /(1+\gamma)$. Using these, we can estimate $V^{\prime}(s)$ in $(9)$ as follows:

$$
V^{\prime}(s) \leq \sqrt{2} K\left|\varphi^{3} \varphi^{\prime \prime}(t)\right| V^{\alpha}(s),
$$

where $\alpha=(\gamma+3) / 2(\gamma+1)>1$. Integrating (10) from $s_{0}=h\left(t_{0}\right)$, we find

$$
V(s) \leq V\left(s_{0}\right)+\sqrt{2} K \int_{s_{0}}^{s}\left|\varphi^{3} \varphi^{\prime \prime}\left(h^{-1}(\tau)\right)\right| V^{\alpha}(\tau) d \tau .
$$

Note that

$$
\int_{s_{0}}^{s}\left|\varphi^{3} \varphi^{\prime \prime}\left(h^{-1}(\tau)\right)\right| d \tau=\int_{t_{0}}^{t}\left|\varphi \varphi^{\prime \prime}(u)\right| d u
$$

which remains finite as $s \rightarrow \infty$. So, by choosing $V\left(s_{0}\right)$ sufficiently small, we can ensure from (11) that $V(s)$ is bounded. In fact, applying Gronwall's inequality to (11) (see e.g. $[20]$ ), we have

$$
V(s) \leq\left\{V\left(s_{0}\right)^{1-\alpha}+(1-\alpha) \int_{s_{0}}^{s}|\lambda(\tau)| d \tau\right\}^{1 /(1-\alpha)}
$$

where $\lambda(\tau)=\sqrt{2} K\left|\varphi^{3} \varphi^{\prime \prime}\left(h^{-1}(\tau)\right)\right| \in L^{1}(0, \infty) . V(s)$ will be bounded if we choose

$$
V\left(s_{0}\right)<\left\{(\alpha-1) \int_{s_{0}}^{\infty} \lambda(\tau) d \tau\right\}^{1 /(1-\alpha)}=L .
$$

Thus, in terms of the solution $y(t)$, we can choose $y\left(t_{0}\right)=0$ and

$$
\frac{1}{2} y^{\prime}\left(t_{0}\right)^{2} \varphi^{2}\left(t_{0}\right)=\frac{1}{2} x^{\prime 2}\left(s_{0}\right)=V\left(s_{0}\right)<L
$$

This establishes the result. 
3. Using the same method as given by Hinton [8], we obtain the same corollary for the sublinear equation, namely,

COROllaRY. Let $0<\gamma<1$ and $\sigma=\gamma+5 / \gamma+3$. If

$$
\int^{\infty}\left|a^{-\sigma}(t) a^{\prime \prime}(t)\right| d t<\infty,
$$

then equation (1) has oscillatory solutions.

Let $a(t)=t^{\beta}$, then conditions (2) and (3) implies that $\beta>-(\gamma+3) / 2$ which is slightly weaker than the condition in Theorem A (cf. [9], [12]) which requires $\beta \geq-(\gamma+3) / 2$. On the other hand, Belohorec $[\mathbf{1}]$ showed that $\int^{\infty} t^{\gamma} a(t) d t=\infty$ if and only if equation (1) is oscillatory. Thus, Belohorec's condition would require $\beta \geq-\gamma-1$ which also implies $\beta>-(\gamma+3) / 2$. For $-(\gamma+3) / 2<\beta<-\gamma-1$, equation $y^{\prime \prime}+t^{\beta} y^{\gamma}=0$ would therefore have both oscillatory and nonoscillatory solutions.

Nehari [16] introduced the notion of proper nonoscillatory solutions, namely those with at least one zero. The absence of such solutions implies that every solution of (1) with a zero must be oscillatory. Indeed, for Theorem A when $\gamma>1$ we can make that stronger assertion. Equations with this stronger property have certain physical meaning in that they describe motions which once pass their rest position must continue passing through this position at all later times (see [18]). However, in the sublinear case, we can only assert that solutions with sufficiently small initial conditions, including both $y\left(t_{0}\right)$ and $y^{\prime}\left(t_{0}\right)$, are oscillatory. This is true both for Theorems A and B. In the latter case see (13). By contrast, Hinton's result of Theorem B when $\gamma>1$, establishes that solutions with sufficiently large $y\left(t_{0}\right)$ are oscillatory. In light of the above discussion, it would be interesting to know whether under assumptions given in Theorem $\mathrm{A}$ when $0<\gamma<1$ and also Theorem B for $\gamma>0$ equation (1) possesses proper nonoscillatory solutions.

\section{REFERENCES}

1. S. Belohorec, Oscillatory solutions of certain nonlinear differential equations of second order, Mat. Fyz. Casopis Sloven. Akad. Vied 11 (1961), 250-255.

2. $\ldots$, On some properties of the equation $y^{\prime \prime}(x)+f(x) y^{\alpha}(x)=0,0<\alpha<1$, Mat. Fyz. Casopis Sloven. Akad. Vied 17 (1967), 10-19.

3. C. V. Coffman and J. S. W. Wong, Oscillation and nonoscillation theorems for second order ordinary differential equations, Funkcial. Ekvac. 15 (1972), 119-130.

4. _ Oscillation and nonoscillation of solutions of generalized Emden-Fowler equation, Trans. Amer. Math. Soc. 167 (1972), 399-434.

5. L. H. Erbe, Oscillation and nonoscillation properties of second order nonlinear differential equations, Proc. Equadiff. Z2 (Wurzburg) 1983 (to appear).

6. L. H. Erbe \& J. S. Muldowney, On the existence of oscillatory solutions to nonlinear differential equations, Ann. Mat. Pura Appl. 59 (1976), 23-37.

7. Math. 12 (1982), 635-642.

8. D. B. Hinton, An oscillation criterion for solutions of $\left(r y^{\prime}\right)^{\prime}+q y^{\gamma}=0$, Michigan Math. J. 16 (1969), 349-352.

9. M. Jasny, On the existence of an oscillatory solution of the nonlinear differential equation of the second order $y^{\prime \prime}+f(x) y^{2 m-1}=0, f(x)>0$, Casopis Pěst. Mat. 85 (1960), 78-83.

10. I. T. Kiguradze, On the oscillatory and monotone solutions of ordinary differential equations, Arch. Math. 1 (Brno) 14 (1978), 21-44. 
11. T. Kura, Oscillation theorems for a second order sublinear ordinary differential equations, Proc. Amer. Math. Soc. 84 (1982), 535-538.

12. J. Kurzweil, $A$ note on oscillatory solutions of the equation $y^{\prime \prime}+f(x) y^{2 n-1}=0$, Casopis Pěst. Mat. 85 (1960), 357-358.

13. M. K. Kwong and J. S. W. Wong, On the oscillation and nonoscillation of second order sublinear equations, Proc. Amer. Math. Soc. 85 (1982), 547-551.

14. Nonoscillation theorems for a second order sublinear ordinary differential equation, Proc. Amer. Math. Soc. 87 (1983), 467-474.

15. __ On an oscillation theorem of Belohorec, SIAM J. Math. Anal. 14 (1983), 474-476.

16. Z. Nehari, On a class of nonlinear second order differential equations, Trans. Amer. Math. Soc. 95 (1960), 101-103.

17. __ A nomlinear oscillation problem, J. Differential Equations 5 (1969), 452-460.

18. _ _ A nonlinear oscillation theorem, Duke Math. J. 42 (1975), 183-189.

19. H. Onose, On Butler's conjecture for oscillation of an ordinary differential equation, Quart. J. Math. Oxford Ser. (2) 34 (1983), 235-239.

20. D. Willett and J. S. W. Wong, On the discrete analogues of some generalizations of Gronwall's inequality, Monatsh. Math. 69 (1965), 362-367.

21. J. S. W. Wong, On the generalized Emden-Fowler equation, SIAM Rev. 17 (1975), 339-360.

22. _ Remarks on nonoscillation theorem for a second order nonlinear differential equation, Proc. Amer. Math. Soc. 83 (1981), 541-546.

China Dyeing Works, LTD., 819 SWire House, Hong Kong

Department of Mathematics, University of Hong Kong, Hong Kong 